\title{
Einführung - Führung im globalen Kontext
}

Die Arbeitswelt ist in den letzten Jahrzehnten erheblich komplexer, unbeständiger und unvorhersehbarer geworden. In diesem Zusammenhang wurde in den USA der Begriff „VUCA World“ geprägt. Das Akronym steht für „Volatile“ (volatil), „Uncertain“ (ungewiss), „Complex“ (komplex) und „Ambiguous“ (mehrdeutig). Für den Erfolg von Organisationen wird dabei die Fähigkeit, sensibel, schnell und angemessen mit Veränderungen im globalen Kontext umzugehen zum entscheidenden Faktor. Damit steigen auch die Anforderungen an Führungskräfte, insbesondere was deren „Vielseitigkeit“ anbelangt: So müssen sie unter anderem mit Mehrdeutigkeit und Unsicherheit in ihrem Arbeitsumfeld umgehen und zugleich handlungsfähig bleiben. Interkulturelle Begegnungssituationen erhöhen dabei zusätzlich die Komplexität. Die Art und Weise, wie Führungskräfte in einem dynamischen Umfeld mit ihren Mitarbeitern, Kunden, Lieferanten und anderen Interessenvertretern aus unterschiedlichen Kulturen interagieren, beeinflusst maßgeblich die Leistungsfähigkeit einer Organisation. Interkulturelle Kompetenz stellt eine wichtige Voraussetzung für den beruflichen Erfolg global agierender Führungskräfte dar. Welche Aspekte sind für das Gelingen von Führung im interkulturellen Kontext von zentraler Bedeutung? Und wie lassen sich Führungskräfte mit den erforderlichen Kompetenzen und Fähigkeiten identifizieren und fördern, um interkulturelle Begegnungssituationen im beruflichen Alltag zu meistern und die Vorteile von Diversität zum Tragen zu bringen?

R. M. Gasteiger et al., Interkulturelle Führung in Organisationen, essentials, DOI 10.1007/978-3-658-12301-7_1 